\section{PTU-127 DOES A DIRECT-TO-SCOPE PATHWAY SIGNIFICANTLY REDUCE TIME TO DIAGNOSIS FOR PATIENTS WITH POSITIVE COELIAC SEROLOGY?}

Douglas Penman*, Rachel Trotter, Adrian Thuraisingam. Wirral University Hospital, Upton, UK

\subsection{6/gutjnl-2018-BSGAbstracts.505}

Introduction NICE Coeliac Disease Quality Standard 134 (QS134) states that: Patients with suspected coeliac disease (CD) should undergo endoscopic (OGD) intestinal biopsy within 42 days of referral and, if confirmed, should receive specialist dietary advice. Given that UK incidence of CD is 19:100,000, our trust would expect 60 new cases of CD per year. In October 2017, a direct-to-scope referral was introduced to streamline the diagnostic pathway. We sought to evaluate the impact of this pathway on the time to histological diagnosis and dietitian review.

Methods All adults referred with positive coeliac serology initiated in primary care from April-September 2017 were compared to those referred following the introduction of the new pathway. Data for the two cohorts was collected from e-case notes using a standard audit tool. This included time from referral to: OGD; confirmation of diagnosis; clinic review; dietician review and vaccination advice.

Results 27 patients (cohort A) were identified in the 6 months prior to October and 17 patients (cohort B) in the following 4 months. In cohort A 2 patients did not attend their appointment and 5 patients underwent OGD prior to referral. From April-September we also identified 10 patients with positive coeliac serology that, to date, have not been referred. The results are shown in table 1 .

\begin{tabular}{llllll} 
Abstract PTU-127 Table 1 & & & & \\
& $\begin{array}{l}\text { mean } \\
\text { (days) }\end{array}$ & range & $\begin{array}{l}\text { mean } \\
\text { (days) }\end{array}$ & range & \\
\hline Result-GP referral & 30 & $7-159$ & 29 & $9-140$ & 0.98 \\
GP referral-OGD & 39 & $4-89$ & 24 & $4-43$ & 0.010 \\
GP referral-clinic & 43 & $13-81$ & 49 & $27-67$ & 0.22 \\
GP referral-confirmation of CD & 68 & $12-$ & 39 & $21-69$ & 0.0012 \\
& & 189 & & & \\
GP referral-dietician review & 104 & $98-$ & 52 & $30-71$ & 0.0035
\end{tabular}

$\begin{array}{lcc}\begin{array}{l}\text { Patients discharged after initial } \\ \text { review }\end{array} & 30.4 \% & 92.3 \% \\ \begin{array}{l}\text { Patients given vaccination advice } \\ \text { Pan }\end{array} & 25.0 \% & 69.2 \%\end{array}$

Conclusions The use of a direct-to-scope pathway significantly reduces time to diagnosis. This change in the pathway allows clinic and dietician review to occur simultaneously, resulting in referral to treatment time within 18 weeks. The only patient in cohort B outside the 6 week target to endoscopy did not tolerate their index OGD and required a repeat endoscopy. Written confirmation of diagnosis now occurs prior to the initial clinic review, helping reduce unnecessary follow-up for patients with uncomplicated CD. We hope continued use of this pathway may also reduce the number of patients with positive serology not referred from primary care.

\section{PTU-128 COMPLEX COLORECTAL POLYP SERVICE IN THE SOUTHEAST REGION: FIRST ANNUAL RESULTS OF A NEW SERVICE}

${ }^{1}$ Giuseppe Preziosi* ${ }^{2}{ }^{2}$ Vasileios Papastergiou, ${ }^{2}$ loannis Stasinos, ${ }^{1} \mathrm{Sun} \mathrm{Mi} \mathrm{Ha}$, ${ }^{1}$ Justin Fegredo, ${ }^{1}$ Nipin Bagla, ${ }^{1}$ Andrew F Muller, ${ }^{1}$ Joseph Sebastian, ${ }^{1,2}$ Zacharias P Tsiamoulos. 'East Kent University Hospitals NHS Foundation Trust, UK ${ }^{2}$ St Marks Hospital, London, UK

\subsection{6/gutjnl-2018-BSGAbstracts.506}

Introduction Recently published Guidelines of the British Society of Gastroenterology (BSG) establish the standards of a Complex Colorectal Polyp (CCP) Service. We assessed the provision of a new CCP Service at East Kent University Hospitals Foundation Trust.

Methods We prospectively recorded our performance for resections of CCPs (low risk $>2 \mathrm{~cm}-4 \mathrm{~cm} /$ high risk $>4 \mathrm{~cm}$ ) from November 2016 to December 2017 and measured it against BSG standards: 1) interval time from referral to resection within 8 weeks, 2) resection types employed, 3) short term outcomes (follow up within 3-6 months) and complications.

Results 105 patients underwent 121 resection procedures (56 males, mean age $70.5 \pm 11.5$ years). The interval time from referral to resection procedure was delayed $>8$ weeks in $36 / 105$ patients (34\%). Polyp resection was completed in one session in 116/121 cases (96\%), > 1 session in 2 cases and on 3 occasions the procedure was abandoned (suspected invasion).

We performed 77 piecemeal-Endoscopic-Mucosal-Resection (pEMR), 8 hybrid pEMR/Endoscopic-Mucosal-Ablation (EMA), 8 hybrid pEMR/Endoscopic-Submucosal-Dissection (ESD), 8 Trans-Anal-Submucosal Endoscopic Resection (TASER), 7 freehand ESD, 2 Laparoscopic-Assisted-Colonoscopy-Polypectomy (LACE) and 11 Serrated Cold Piecemeal Emr (SCOPE). Median overall polyp size was $3 \mathrm{~cm}$ (range 2/12). For 13/38 polyps with high-risk features (median size of $5 \mathrm{~cm}$, range 4 12), otherwise destined for tertiary referral or surgery, we selected more endo-surgical and/or en-bloc resections: $(\mathrm{TASER}=7 / \mathrm{ESD}=4 / \mathrm{LACE}=2)$.

12 Polyps proved to have malignant features (11 within the high-risk group), all discussed at Colorectal MDM: 6/12 underwent colectomy, 2/12 local radiotherapy (T1 $>1 \mathrm{~mm}$ +adverse histological features/patient preference) and 4/12 endoscopic surveillance (T1 $<1 \mathrm{~mm}$, no adverse histological features). In total, $7 / 105(6.6 \%)$ patients had surgery (6 malignant/1 benign polyps).

Of 98/105 patients treated: 41 (42\%) did not have followup within 6 months, $31(30 \%)$ are scheduled for follow-up and 27 (28\%) had timely follow-up with a low (range 3$10 \mathrm{~mm})$ recurrence rate $(4 / 27,13.8 \%)$, easily treated. No perforations or mortality were recorded. Four cases of delayed bleeding (3.8\%) were documented: endoscopy was required in 2 for clipping.

Conclusion Our results demonstrated a safe and effective provision of a CCP service $(96 \%$ complete excision in a single visit), as a result of a synergy between gastroenterologists and surgeons. However, we noticed an inadequate booking process, currently addressed by implementing new CCP pathways and setting up a CCP MDM. 